\title{
Extension and Implementation of Curve
}

\author{
Honglin Li
}

School of Information Engineering, Qujing Normal University, Qujing Yunnan 655011, China lihonglin28286@sina.com

Keywords: Curve; Similar bezier; Parameter

\begin{abstract}
In this paper, the positive leaf lines and the positive leaf bow are generated by the transformation of curve. For example, the bezier curve. It doesn't change the basic properties and the control points of the berzier curve, and then we can extend it.The shape of the curve is changed by adjusting the factor, which is helpful for the design of complex curves and surfaces. It has a certain reserch value.
\end{abstract}

\section{Introduction}

The orbit of the satellite, the trajectory of the missile, the shape of the car and the airplane, the pattern and the pattern design in the daily life, can not be separated from the description of the curve and surface and the drawing [1]. The curve is the foundation of the surface, which we often encounter and it plays an important role in our life. Through the curve transformation, can generate a variety of beautiful graphics, is conducive to the realization of the project, parts processing, etc..

\section{The Positive Leaf Line and the Bow}

The Positive Leaf Line. The positive leaf line is a curve similar to the shape of a plant leaf

[2].The parameter equation of positive leaf line is as follows:

$$
\left\{\begin{array}{l}
r=a \sin (n \theta) \\
x=r \cos (\theta) \\
y=r \sin (\theta), \text { in this }
\end{array}\right.
$$
equation, $0<\theta<2 \pi$, $a>0, n=2,3,4, \ldots \ldots$

A beautiful pattern can be generated by a positive line. When a takes $80, n$ takes 2,4 respectively, as shown in Fig. 1.

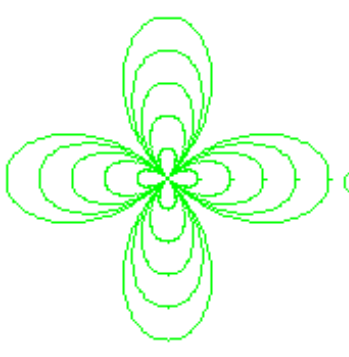

$\mathrm{n}=2$

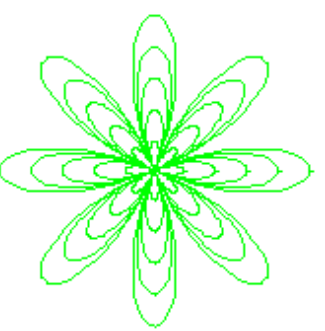

$\mathrm{n}=4$

Figure 1. The Positive Leaf Line

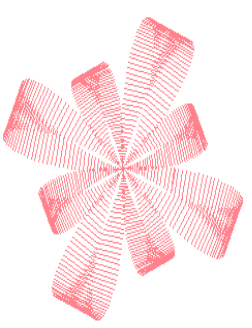

Figure 2. Positive Leaf Bow

The Positive Leaf Bow. The bow, as its name implies, is similar to the design of a bow [2]. After the establishment of two-dimensional coordinates $\mathrm{x}$, $\mathrm{y}$ function and the use of the transformation of the draw. Draw the line point of the $\mathrm{X}$ coordinates, $\mathrm{y}$ coordinates according to a function of the law of rotation changes, that is the bow pattern, as shown in Fig. 2. 


\section{Bezier Curve}

In various curves, the Bezier curve is often used. Bezier curve is one of the most important research contents of computer aided geometric design. It is very important in engineering design. The Bezier curve is generated by the de Casteljan algorithm. The algorithm uses a series of point P0, $\mathrm{P} 1, \mathrm{P} 2$... A well-defined value of $\mathrm{P}(\mathrm{T})$ is constructed for each $\mathrm{t}$ value between 0 and 1 . Therefore, this algorithm provides a series of methods for generating curves.If we Change these points, and the curve also is changed [3].

Bezier curve definition: given space $n+1$ point position vector $\mathrm{Pi}(\mathrm{i}=0,1,2, \ldots \mathrm{n})$, the interpolation formula of the coordinates of each point on the Bezier parameter curve is:

$$
P(t)=\sum_{i=1}^{n} P_{i} B_{i, n}(t), t \in[0,1]
$$

In the formula, $\mathrm{Pi}$ constitutes the characteristic polygon of the Bezier curve, $\mathrm{Bi}, \mathrm{n}(\mathrm{t})$ is a $\mathrm{n}$ Bernstein basis function:

$$
B_{i, n}(t)=C_{n}^{i} t^{i}(1-t)^{n-i}=\frac{n !}{i !(n-i) !} t^{i}(1-t)^{n-i} \quad(i=0,1, \ldots, n)
$$

Connect two adjacent control points $\mathrm{Pi}, \mathrm{Pi}+1(\mathrm{i}=0,1), \ldots, \mathrm{n}-1$, will get a $\mathrm{n}$ edge line $\mathrm{P} 0, \mathrm{P} 1, \mathrm{P} 2, \ldots$ $\mathrm{Pn}$, the $\mathrm{n}$ edge of a line called Bezier control polygon, referred to as Bezier polygon, as shown in Figure 3 , which is drawn by the dashed line.

\section{Quasi Bezier Curve}

The Bezier curve has good geometric properties and can be used to express the free curve and surface. It is very important to extend the Bezier curve without changing the basic properties of the Bezier curve. At present, the extension of the Bezier curve is more, for example: $n$ Bezier curve extension [4], for the extension of the cubic, the quartic Bezier curve [5][6]. After the expansion, the shape of the curve and surface can be adjusted more flexibly under the condition that the control points are not changed. This extended Bezier curve is called the Quasi Bezier curve.

A Quasi Bezier Curve with a Parameter. We can Extend the Bernstein basis function as follows:

$$
\begin{aligned}
& B_{i, n}[f(t)]=C_{n}^{i}[f(t)]^{i}[1-f(t)]^{n-i}, \text { among them, } \mathrm{F}(\mathrm{T}) \text { is a continuous function on } \mathrm{t} \\
& f(t)=\frac{t^{\alpha}+t}{2}, \alpha>0 \text {, } \alpha \text { is called the adjustment factor of Bezier curve ; when } \alpha=1 \text {, quasi Bezier } \\
& \text { [7]. }
\end{aligned}
$$
curve is the Bezier curve; $f(t) \in[0,1]$.

Definition: $\mathrm{Bi}, \mathrm{n}[\mathrm{f}(\mathrm{t})]$ is the quasi Bernstein polynomial. $\mathrm{Bi}, \mathrm{n}[\mathrm{f}(\mathrm{t})]$ as the basis function of the curve is called the quasi Bezier curve.

Quasi Bezier Curves with Two Parameters. The Bernstein basis functions are generalized as follows:

$$
\begin{aligned}
& B_{i, n}[f(t)]=C_{n}^{i}[f(t)]^{i}[1-f(t)]^{n-i} \text {, among them, } \mathrm{F}(\mathrm{T}) \text { is a continuous function of } \mathrm{t} . \\
& f(t)=\frac{(u-1) t^{\alpha}+t}{u}, \alpha>0, u \geq 2 \text {, when } \alpha=1 \text {, quasi Bezier degenerate into Bezier curve }
\end{aligned}
$$
[8].

Whether it takes a parameter or a quasi Bezier curve with two energy numbers, not only includes almost all the good properties of the Bezier curve, but also overcomes some defects of the Bezier curve. For example, the same point: (1) endpoint properties: Bezier curve, the starting point and end point of quasi Bezier curve and the corresponding control polygon starting point, end point of coincidence; (2): the starting point and end point of tangent vectors and the corresponding control polygon, and the starting point and end point of the tangent direction of the first side and the control 
polygon and finally an edge direction etc.. Different points: the number of Bezier curve is determined by the number of control points; the number of quasi Bezier curve is determined by the control points and the regulator can achieve one order and two order continuous curvature and good continuity, is conducive to the curve matching. In particular, the Bezier curve with two parameters, through the adjustment of the adjustment factor and the change of the parameter $\mathrm{u}$, has greater freedom and flexibility.For example, with six vertices $(50,50),(150,160),(200,180),(240,80)$, $(260,40),(300,200)$, which generated Bezier curve and the quasi Bezier curve $(\alpha=0.4)$ with a parameter are shown in Fig. 3 and Fig. 4.

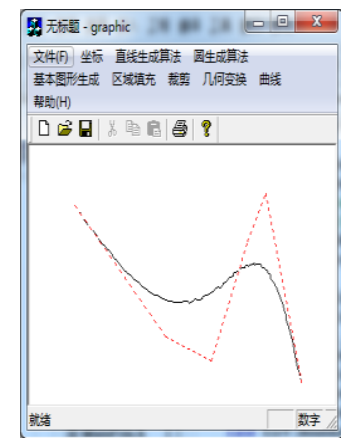

Figure 3. BezierCurve

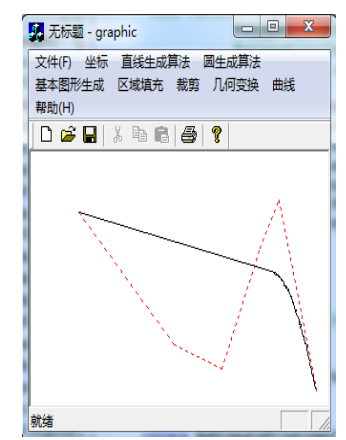

Figure 5. $\quad \alpha=0.4, \mathrm{u}=4$ )

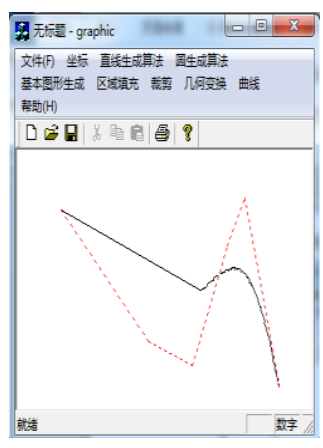

Figure 4. A Parameter

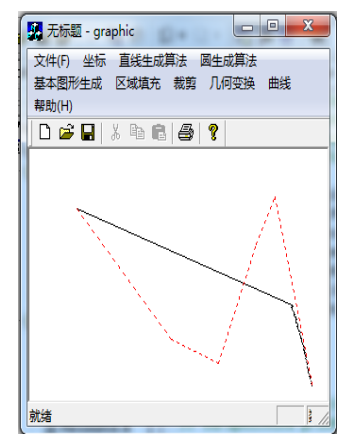

Figure 6. $\alpha=0.4, \mathrm{u}=9$

Quasi Bezier curve with two parameters, when the adjustment factor $\alpha=0.4$, u were taken at 4 and 9, respectively, as shown in Fig.5 and Fig. 6.

Comparison. Six vertices are used to control the vertices of the polygon, and the Bezier curve, the Bezier curve with one parameter and the quasi Bezier curve with two parameters are generated. When the adjustment factor $\alpha=1$, the quasi Bezier curve degenerates to Bezier curve. That is, using the three algorithms to generate the same Bezier curve, as shown in Fig. 3, the operating speed as shown in Table 1.

Table 1 Run time of the three algorithms [S]

\begin{tabular}{|c|c|c|c|}
\hline & Bezier Curve & With a parameter $(\alpha=1)$ & $\begin{array}{c}\text { With two parameter } \\
(\alpha=1, \mathrm{u}=9)\end{array}$ \\
\hline \multirow{2}{*}{ Run times } & 0.000825 & 0.001386 & 0.001302 \\
$(3$ times ) & 0.000858 & 0.001373 & 0.001312 \\
& 0.000637 & 0.001336 & 0.001325 \\
\hline
\end{tabular}

As can be seen from the table, when the same Bezier curve is generated, the quasi Bezier curve with parameter is longer than that without parameter. 


\section{Concluding Remarks}

Through the curve transform can generate beautiful graphics; and expansion of the wide application of Bezier curve in life, the Bezier curve between the expanded curve and the traditional extended Bezier curve with parameter values can adjust the parameters to change the shape of the curve without changing the control points, this method is simple and fast, plays a very important role in curve and surface design.

\section{Reference}

[1] Y.Y. Chen, R.ZH. Zhang and D. Wu: Practical Techniques of Computer Graphics (Tsinghua University press, China 2007. (In Chinese)

[2] Q.F. He: Principles and Algorithms of Computer Graphics (Tsinghua University press, China 2010. (In Chinese)

[3] F.S.Hill, JR: Computer Graphics (Tsinghua University press, China 2006).

[4] H.Er.M.J.H.J. DaMaiLiHan: Bezier Curve and its Application (MS.,HeFei University of Technology, China 2014). (In Chinese)

[5] R. Qiu, H.J. Hang and J.CH. Pan: Computer Engineering and Application, Vol. 50 (2014) No.20, p.158-162. (In Chinese)

[6] H.J. Hang, J. Yu, W.G. Li: Computer Engineering and Application, Vol. 46 (2010) No.31, p.178-180. (In Chinese)

[7] ZH. Li, M. Huang and D.F. Han: Computer Engineering and Applications, (2003) No.2, p.15-17. (In Chinese)

[8] H.Y. Xi,G.C. Zhang: Computer Science, Vol. 41 (2014) No.11a, p.100-102. (In Chinese) 